\title{
Premiers inventaires de la diversité des macroinvertébrés aquatiques du lac Vert, Goma/Nord- Kivu, République Démocratique du Congo.
}

\author{
Cubaka Kabagale Alfred1', Cirimwami Bahimirwe Légrand², Bora Uzima Henri³, Lwambo Kabolo \\ Jospin $^{3}$ et Baguma Balagizi Gabriel ${ }^{1 *}$ \\ 1. Laboratoire de Physiologie Végétale et de Microbiologie Appliquée, Faculté de Sciences, Université \\ Officielle de Bukavu, LPVMA-UOB, B.P 570 Bukavu, Sud-Kivu, République Démocratique du Congo \\ 2. Département de l'Environnement, Faculté de Sciences, Université du Cinquantenaire de Lwiro, UNI-50, \\ Sud-Kivu, République Démocratique du Congo \\ 3. Option de Biologie Appliquée, Département de Biologie-Chimie, Section Sciences Exactes, Institut \\ Supérieur Pédagogique de Goma, ISP/GOMA, Nord-Kivu, République Démocratique du Congo. \\ 4. Auteur en correspondance : gabrielbalagiz@gmail.com
}

Original submitted in on $6^{\text {th }}$ June 2019. Published online at www.m.elewa.org/journals/ on $31^{\text {st }}$ August 2019 https://dx.doi.org/10.4314/jab.v140i1.6

\section{RESUME}

Objectif : Cette étude vise l'inventaire des macroinvertébrés du Lac Vert pour faciliter sa conservation et le suivi régulier des changements que subit ce lac, sa biodiversité et ses services écosystémiques surtout parce que les macroinvertébrés sont des véritables bioindicateurs des changements.

Méthodologie et Résultats: Les macroinvertébrés aquatiques ont été récolté par la méthode homme surface (Touzin, 2008; Melhaoui et Berrahou, 2009). à l'aide d'un filet troubleau dans 4 sites choisis en fonction des activités anthropiques, du type de substrat et de la végétation riveraine. Dix (10) Ordres répartis en 22 Familles et 26 Genres avec 1635 individus ont été inventoriés. Les analyses statistiques ont montré que les indices de Shannon et d'Equitabilité n'ont pas de différence significative (Kruskal-Wallis, ddl $=3$, Chi $^{2}=4,15$ et $p$-value $=0,25$ ) entre les sites mais les sites Rutanda-Busara différent des autres en terme de Richesse Spécifique ( $p$-value $=0,005)$.

Conclusion et application des résultats: Le Lac Vert regorge une diversité des macroinvertébrés aquatiques importante par rapport aux lacs Kivu, Edouard, Tanganyika et Albert. Une abondance des Coléoptères et Mollusques a été trouvée rapprochée par une flore importante, cas des macrophytes et du phytoplancton. Les activités anthropiques sont les principaux facteurs influençant la diversité des macroinvertébrés dans cet écosystème. Cette étude ouvre une question scientifique sur différents aspects de la recherche sur la taxonomie, l'écologie et structure fonctionnelle des invertébrés aquatiques de cet écosystème pour une étude approfondie dans ce domaine. Ceci ferait de ce lac un bon jardin zoologique et botanique au bienêtre de l'humanité.

Mots clés : Macroinvertébrés Aquatiques, Shannon, Equitabilité, Richesse Spécifique, Lac Vert. 


\section{ABSTRACT}

Objective: This study aims to inventory the macroinvertebrates of Lac Vert to facilitate its conservation and regular monitoring of the changes that this lake, its biodiversity and its ecosystem services undergoe mainly because macroinvertebrates are absolute bioindicators of changes.

Methodology and Results: Aquatic macroinvertebrates were collected by the human surface method using a trickle net in 4 sites chosen according to human disturbance, type of substrate and surrounding vegetation. Ten (10) Orders divided into 22 Families and 26 Genera with 1635 individuals were inventoried. Statistical analyses showed that the Shannon and Equitability indices have no significant difference (Kruskal-Wallis, $\mathrm{ddl}=3 \mathrm{Chi}^{2}=4.15$ and $\mathrm{p}$-value $=0.25$ ) between the sites but the sites Rutanda-Busara different from others in terms of specific wealth ( $p$-value $=0.005$ ).

Conclusion and application of results: The Lac Vert lake has a large diversity of aquatic macroinvertebrates compared to lakes Kivu, Edouard, Tanganyika and Albert. An abundance of Coleoptera and Molluscs was found to be close to a flora composed of macrophytes and phytoplankton. Human disturbance activities are the main factors which act on the diversity of macroinvertebrates in this ecosystem. This study opens a scientific question on different aspects of the research on the taxonomy, ecology and functional structure of aquatic invertebrates of this ecosystem for an in-depth study in this field. This would make this lake a good zoological and botanical garden for the welfare of humanity.

Keywords: Aquatic Macroinvertebrates, Shannon, Equitability, Specific Wealth, Lake Vert .

\section{INTRODUCTION.}

La République Démocratique du Congo (RDC) dispose des plaines inondées et lacs couvrant environ $56080 \mathrm{~km}^{2}$ soit $3,5 \%$ de la superficie nationale et ayant un potentiel halieutique considérable (MECNT, 1997). L'un de groupes les plus étudiés est les macroinvertébrés aquatiques qui occupent un vaste ensemble de niche écologique dans l'environnement aquatique (Véronique, 2014). Ils participent au processus de décomposition qui conduit au recyclage des nutriments (Bournaud et al., 1980 ; Rosenberg \& Resh, 1993 ; U.S. EPA, 2002 ; CVRB, 2006 ; Véronique, 2014), sont des bioindicateurs de la qualité biologique des eaux et état écologique des écosystèmes (Micha et al., 1982), sont des régimes alimentaires des poissons et amphibiens (Gnohossou, 2006; WWF, 2007; Touzin, 2008; Pollabauer et al, 2010; AFNOR, 2010). L'écologie, la biologie et la systématique des différents taxons des macroinvertébrés aquatiques ont été au cœur des différents chercheurs dans les eaux douces durant quatre décennies (; MDDP, 2008 ; Grant, 2016). Dans l'Est de la RDC, les investigations sont fragmentaires. Néanmoins, l'effet des paramètres tels que la saison, les activités anthropiques et le cycle limnologique influencent la diversité des macroinvertébrés aquatiques de différentes rivières et marais du bassin du lac Kivu tel que relevé par (Ngera et al. 2009a; Zirirane et al., 2014 ; Ndakala et al., 2015; Irenge, 2012). Ceci contribuent à la perturbation de l'habitat aux détriments des macroinvertébrés aquatiques (Cummins, 1975; Léveque, 1996; Leclerqu, 2001; Touzin, 2008; Tachet et al., 2009; Véronique 2014). Les travaux de Palmer et al. (1997) ont montré que les macroinvertébrés aquatiques sont fortement influencés par la nature du substrat qui peut conduire à la répartition des peuplements sous forme mosaïque. Ces aspects n'ont jamais été abordés dans les écosystèmes du lac Vert. Les macroinvertébrés aquatiques du lac Vert, nous intéresse en tant que tels car encore inconnus. L'une de raison de cette étude est que certains groupes des macroinvertébrés sont des bio-indicateurs de la qualité écologique pouvant permettre le monitoring de l'impact des activités anthropiques sur cet écosystème (Véronique, 2014). Par exemple, une diversité élevée en macroinvertébrés fait souvent preuve d'une bonne source alimentaire des poissons d'un écosystème aquatique (Tachet et al., 2009). Les activités anthropiques entre autres la lessive, la vaisselle, la baignade, l'agriculture et le déboisement observé dans les sites d'échantillonnages permettent de 
différencier la richesse et abondances des macroinvertébrés aquatiques selon les sites tels que relevé par Ngera et al., (2009) lors d'une étude similaire dans la région du Kivu. La localisation de ce lac dans un cratère volcanique éteint laisse penser qu'il peut regorger une biodiversité aquatique particulière comparativement aux autres écosystèmes

\section{MATÉRIEL ET MÉTHODES}

Milieu d'étude : Le lac Vert est l'un de lac des cratères issu de la séparation des plaques tectoniques dans le rift albertin, une ancienne réserve naturelle forestière lac Vert (ICCN, 2014), un cratère volcanique éteint depuis 1923 (Kwetu et al., 2016). II occupe 470.000 mètres carrés soit $41,7 \%$ de la superficie totale du cône (ICCN, 2014). Sans communication directe avec une rivière, les eaux du lac Vert, météoritiques comme pour la plupart des lacs de cratère, sont vertes en apparence, probablement en raison de la présence d'algues vertes. Ses eaux, situées à environ $1463 \mathrm{~m}$ d'altitude, avaient été repoussées, sur environ $7.500 \mathrm{~m}^{2}$ soit 2,9\% de la superficie totale d'eaux du lac (Muhindo, 2014), par une coulée de lave venant probablement du Nyiragongo au Nord et qui entoura le reste du cône (Kwetu et al., 2016). Le type de climat est de subéquatorial d'altitude, et le sol est volcanique. Ce lac est dans la boucle Ouest du système de rift est-africain qui comprend les lacs Kivu, Tanganyika, Edouard, Albert et Georges. Au lac Vert, l'équilibre entre les apports et pertes en eaux, et leurs répartitions dans le temps est dû essentiellement aux pluies et dans certains cas aux nappes phréatiques. Les pertes peuvent provenir des émissaires, de l'évaporation et de l'infiltration. Les études scientifiques conduites dans cette réserve mettent en évidence une dégradation rapide de ses composantes biologiques (ICCN, 2014); prononcé par l'exploitation de carrière de sable, la pêche, l'agriculture, la lessive, le lavage, et la baignade aquatiques de la région. Cette étude veut étudier la diversité alpha et l'abondance des macroinvertébrés de ce site ainsi que faire une brève comparaison avec d'autres résultats obtenus dans d'autres écosystèmes aquatiques n'étant pas dans les mêmes conditions écologiques que le Lac Vert.

influenceraient la composition de la diversité aquatique et l'habitat de cet écosystème fragile.

Caractérisation des sites d'échantillonnage : Quatre sites ont été choisis en raison du type de substrat et des activités anthropiques (MDDP, 2008). Le site de Busara est caractérisé par les roches, les détritus et les macrophytes. II est le site témoin en raison de son couvert végétal presque naturel et un faible taux d'activités anthropiques (seulement l'agriculture). Les 3 autres sites Katorwa, Nvomo et Rutanda ont les mêmes influences des activités anthropiques (agriculture, lessive, baignade, vaisselle). Dans les sites Katorwa et Nvomo on y rencontre en commun des vases et boues comme substrat. Au lac Vert, l'activité commune est la pêche à la ligne et au filet maillant dormant pour la capture des espèces de poissons. Dans sa rive, la végétation est composée de Commelina diffusa, Maessa lanceolata, Ficus exasperata, Dracaena afromontana, Cynodon dactylon, Setaria barbata, Lantana camara, Phytolacca dodecandra, Ricinus communis, Typha sp, Typha latifolia, Digitaria vestida, Pennisetum trachyfolium, Cynodon plectostachys, Pilea microphilla, Ageratum conisoides, Crassocephalum montuosum... (Mushengezi et al. 2018 données non publiées) qui joueraient une importance dans la régulation de la température et la rétention de polluant (Vernaux, 1973; MDDP, 2008). La figure 1 localise le lac Vert et nos sites d'échantillonnages. 


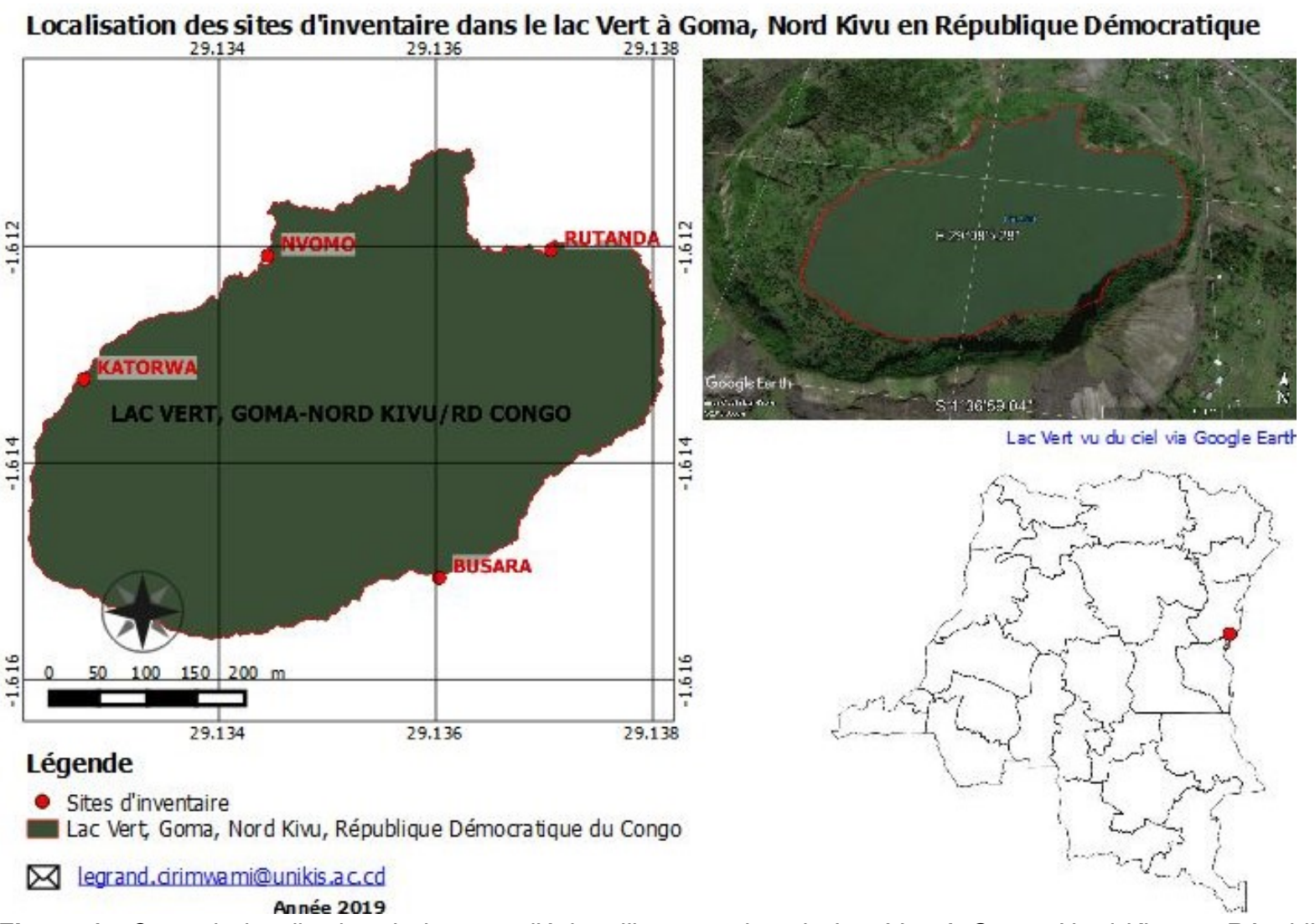

Figure 1: Carte de localization de la zone d'échantillonnage dans le Lac Vert à Goma, Nord Kivu en République Démocratique du Congo.

Récoltes des macroinvertébrés aquatiques du lac Vert: Le prélèvement des macroinvertébrés aquatiques a été effectué au lac Vert à l'aide d'un filet troubleau de maille $250 \mu \mathrm{m}$, par la méthode hommesurface. Elle consiste à entrer au bord des berges du lac à l'aide des bottes et puis manipuler le filet par un coup de pieds (Touzin, 2008; Melhaoui et Berrahou, 2009). L'échantillonnage s'est effectué à des endroits accessibles avec une profondeur moyenne de $1 \mathrm{~m}$ qui a permis d'obtenir une diversité en macroinvertébrés aquatiques caractéristiques des prélèvements réalisés avec une détermination systématique jusqu'au niveau de l'ordre, de la famille et le genre selon les groupes (Grant, 2016). Par endroits, les substrats inférieurs (roches, graviers, macrophytes, boue) végétation et tous les débris flottants ou submergés étaient explorés avec les mains pour y déceler les spécimens qui pourraient y être accrochés (Touzin, 2008 ; Khettar et al., 2013 ; Reyjol, 2013). Les différentes récoltes ont été chaque fois versées sur un tamis de $500 \mu \mathrm{m}$ de maille pour un pré-trien séparant les matières grossières et la récupération des spécimens à l'aide d'une pince entomologique (AFNOR, 2010). Après fixation à l'éthanol dilué à 70\% (Touzin, 2008; AFNOR, 2010), les spécimens récoltés ont été conservés dans des bocaux appropriés, préalablement étiquetés par site. Ces bocaux ont été alors transportés au Laboratoire de Physiologie Végétale et de Microbiologie Appliquée de l'Université Officielle de Bukavu (LPVMA/UOB) pour les analyses finales.

Tri et identification des macroinvertébrés aquatiques au laboratoire: Au LPVMA/UOB, les échantillons conservés dans des bocaux étiquetés par site ont été rincé abondamment sur une série de tamis de mailles de tailles décroissantes (5 à $0,2 \mu \mathrm{m}$ ) (Moussa et al., 2014) afin d'éliminer au maximum le substrat fin restant et les éléments grossiers (MDDP, 2008). Le contenu de tamis était ensuite versé sur un plateau pour le groupement des taxons effectué à l'aide d'une pince entomologique. L'observation et identification ont été réalisé à l'aide d'une loupe binoculaire de marque Forty American Optical Corporation, et des clés d'identification de Tachet et al. (2010), Melhaoui et al. (2009), Micha \& Noiset (1992), Tachet et al. (2009), Leclercq et al., (2010), Nieser (2004), Epler (2006) et Verneaux (1973). Le but de 
l'identification est de déterminer la qualité des classes systémiques présentes dans l'échantillon (diversité) et la présence des groupes taxonomiques les plus sensibles (De Pauw et al, 1983).L'identification a été réalisée sur les larves, les nymphes et les adultes des taxas considérés avec comme unité taxonomique le genre.

\section{Analyse statistique et traitement des données}

Indices écologiques : La fréquence d'occurrence $(F)$ d'un taxon est le ratio entre le nombre d'échantillon $(\mathrm{Pa})$ d'un site où le taxon est présent par le nombre total $(P)$ d'échantillon.

$$
\mathrm{F}=\frac{P_{a}}{P} \times 100
$$

Quatre groupes sont ainsi définis (Dajoz, 1982): le premier concerne les taxons " très fréquents » avec $F$ $\geq 50 \%$, le deuxième groupe correspond aux individus " fréquents » avec $25 \% \leq \mathrm{F}<50 \%$, les taxons rares forment le troisième groupe avec $\mathrm{F}<25 \%$. Dans cette étude de comparaison de retenues, un quatrième groupe est aussi défini : il s'agit des individus absents

\section{RESULTATS}

Aperçu sur la diversité des macroinvertébrés aquatiques du lac Vert: Dans cette étude, nous avons inventoriés 1635 individus répartis en 26 genres, 20 familles et 10 ordres. Les genres les plus abondants comprennent le genre Radix (426 individus, soit 26\%), Stagnicola (160 individus, soit $10 \%$ ) et Neoplea (118 individus, soit 7\%). Les autres genres (Tabanus, Capioneura, Eristalis et Nepa) ont chacun 1 à 7 individus. Pour les familles, la famille de Coenagrionidae est la mieux représentée avec 3 genres (13\%), la famille de Limnaeidae a 2 genres (37 avec $\mathrm{F}=0 \%$. La diversité spécifique $\mathrm{H}^{\prime}$ intègre le nombre total d'espèces présentes dans une communauté considérée avec la fréquence relative des espèces présentes dans cette même communauté (Ramade et Graw-Hill, 1990). L'équitabilité J (ou régularité) d'un échantillon représente le rapport de la diversité spécifique observée à la diversité maximale théorique pouvant être obtenue avec le même nombre d'espèces (Dajoz, 1982). Comme indices écologiques nous avons calculé les fréquences d'occurrences et les indices de diversités (de Shannon Weaver et d'Equitabilté de Pielou).

Analyses statistiques: Le logiciel Past (PAleontological STatisctics, Version 2.16, Hammer et al., 2001) a été utilisé pour construire le dendrogramme de classification hiérarchisée permettant la discrimination des retenues d'eau. La distance euclidienne est utilisée comme distance d'assemblage. Les différents indices écologiques ont été calculés grâce à Past et $\mathrm{R}$ (Millot, 2009).

$\%$ ) et enfin celle de Libelliidae a également 2 genres (soit $8 \%$ ), les autres familles ont moins de 1 genre chacune. L'ordre de Basamatrophore a été plus représenté en termes du nombre individus soit $36 \%$, suivi des Hétéroptère et Odonate avec respectivement $24 \%$ et $23 \%$. Les Acheta, Oligochète et les Plécoptères étaient moins abondants ayant en général moins de $7 \%$. Dans le tableau 1 nous reprenons les chiffres clés des abondances de différents sites selon les principaux taxons concernés par cette étude (individu, genre, famille et ordre).

Tableau 1 : Abondance relative des macroinvertébrés aquatiques du lac Vert

\begin{tabular}{lllllllllllll}
\hline Sites & \multicolumn{3}{c}{ BUSARA } & \multicolumn{3}{c}{ KATORWA } & \multicolumn{3}{c}{ NVOMO } & \multicolumn{3}{c}{ RUTANDA } \\
\cline { 2 - 13 } Ordres & Fam. & Gen. & Ind. & Fam. & Gen. & Ind. & Fam. & Gen. & Ind. & Fam. & Gen. & Ind. \\
\hline Basomatrophora & 1 & 2 & 163 & 1 & 2 & 143 & 1 & 2 & 166 & 1 & 2 & 114 \\
Neogastropoda & 1 & 1 & 5 & 1 & 1 & 5 & 1 & 1 & 11 & 1 & 1 & 12 \\
Odonata & 4 & 7 & 162 & 4 & 7 & 81 & 4 & 7 & 103 & 2 & 3 & 24 \\
Diptera & 2 & 2 & 9 & 2 & 2 & 69 & 2 & 2 & 20 & 3 & 3 & 4 \\
Oligocheta & 1 & 1 & 3 & 1 & 1 & 4 & 1 & 1 & 5 & 1 & 1 & 3 \\
Heteroptera & 7 & 8 & 169 & 7 & 7 & 90 & 7 & 8 & 90 & 7 & 8 & 52 \\
Coleoptera & 2 & 3 & 38 & 2 & 3 & 15 & 2 & 3 & 12 & 2 & 3 & 9 \\
Plecoptera & 1 & 1 & 3 & 1 & 1 & 2 & 0 & 0 & 0 & 0 & 0 & 0 \\
Hydracaria & 1 & 1 & 20 & 1 & 1 & 3 & 1 & 1 & 7 & 1 & 1 & 8 \\
Acheta & 0 & 0 & 0 & 0 & 0 & 0 & 0 & 0 & 0 & 1 & 1 & 11 \\
Total & $\mathbf{2 0}$ & $\mathbf{2 6}$ & $\mathbf{5 7 2}$ & $\mathbf{2 0}$ & $\mathbf{2 5}$ & $\mathbf{4 1 2}$ & $\mathbf{1 9}$ & $\mathbf{2 5}$ & $\mathbf{4 1 4}$ & $\mathbf{1 9}$ & $\mathbf{2 3}$ & $\mathbf{2 3}$ \\
\hline
\end{tabular}

Légende : Fam = Famille, Gen $=$ Genre, Ind = Individus 
L'abondance des macroinvertébrés en termes du nombre total des individus n'est pas la même quand les 4 sites sont comparés $\left(\mathrm{Chi}^{2}=137,46 ; \mathrm{ddl}=3 ; \mathrm{p}<\right.$ 0,0001). Le site de Busara a plus d'individus de macroinvertébrés comparativement aux trois autres sites. En termes du nombre total des familles, les quatre sites ne présentent aucune différence significative $\left(\mathrm{Chi}^{2}=0,05 ; \mathrm{ddl}=3 ; \mathrm{p}=0,997\right)$ de même que quand on les compare en termes du nombre total des genres $\left(\mathrm{Chi}^{2}=0,19 ; \mathrm{ddl}=3 ; p=0,979\right.$ ).

Indice de diversité et richesse spécifique dans les différents sites: L'indice de diversité de Shannon ne varie pas d'un site à un autre (Kruskal-Wallis, $\mathrm{ddl}=3$, $\mathrm{Chi}^{2}=7,47$ et $p$-value $=0,058$ ). De même, l'indice de l'équitabilité de Piélou n'a pas montré de différence significative entre les quatre sites inventoriés (KruskalWallis, ddl $=3, \mathrm{Chi}^{2}=4,15$ et $p$-value $=0,25$ ). Pour la richesse spécifique exprimée en termes du nombre de genres par site, une différence significative a été trouvée (Kruskal-Wallis, ddl $=3, \mathrm{Chi}^{2}=11,66$ et $p$-value $=0,0087$ ). La comparaison site par site a montré l'existence d'une différence significative entre Rutanda et Busara ( $p$-value $=0,005)$ alors que les autres sites n'ont pas montrés de différence significative. Le site de Busara est plus diversifié en termes de la richesse spécifique exprimée par le nombredes genres. La figure 3 présente les indices de diversité de Shannon, d'Equitabilité de Piélou et la richesse spécifique des macroinvertébrés aquatiques selon les sites d'échantillonnage explorés au lac Vert.
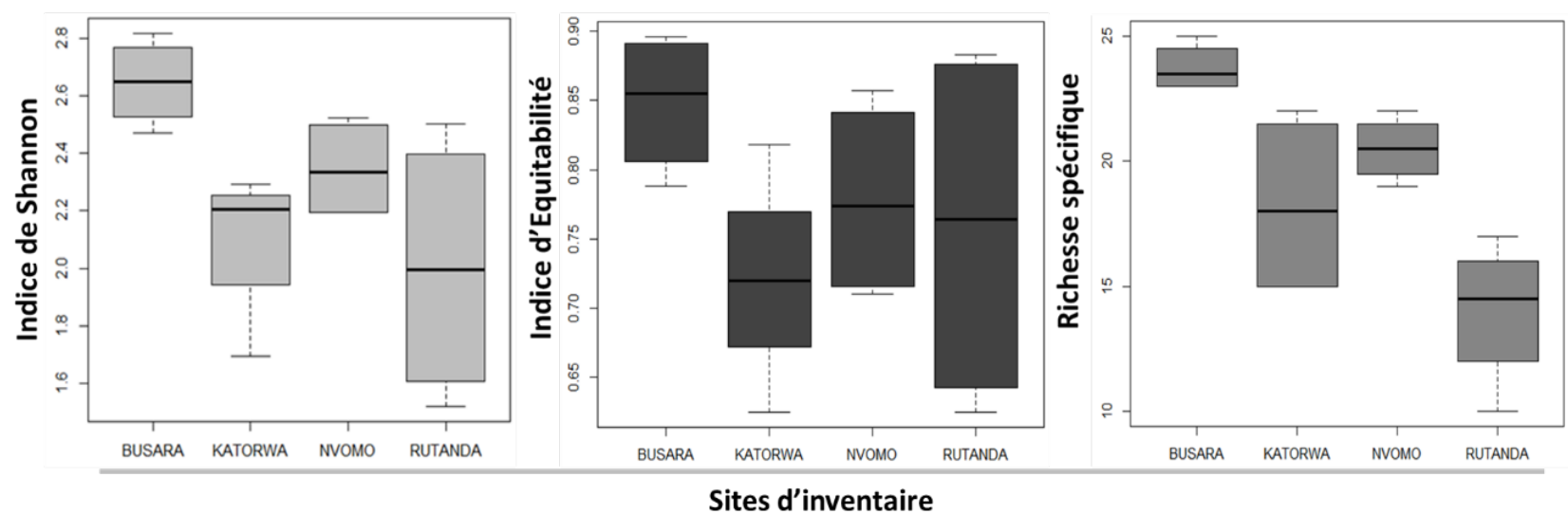

Sites d'inventaire

Figure 3 : Indice de diversité de Shannon, Equitabilité de Piélou et la richesse spécifique dans les quatre sites d'inventaires.

Classification hiérarchique des sites du lac Vert basée sur la similarité des genres des macroinvertébrés aquatiques: La classification hiérarchique discrimine le site de Busara (moins anthropisé) de trois autres sites qui sont plus anthropisés. L'axe 1 et 2 de cette classification exprime $85,1 \%$ de la variation totale observée. De ce fait, le site de Busara constitue une communauté à part entière qui est différente de trois autres sites d'inventaires (Rutanda, Katorwa et Nvomo) qui avaient montré un degré de menaces d'ordre anthropique plus avancé.

Fréquence d'occurrence des familles et genres des macroinvertébrés aquatiques du lac Vert: Sur un total des 20 familles récoltées au lac Vert, seules 5 familles des insectes sont très fréquentes dans le site Busara avec une fréquence d'occurrence > à $50 \%$ dont la famille de Coenagrionidae, d'Aeshnidae, de Belostomatidae, de Disticidae, de Capniidae, et d'Areneidae. La famille de Tabanidae est seulement très fréquente dans le site Katorwa. Le site Rutanda le plus anthropisé a une fréquence d'occurrence très faible ( $F=0 \%$ ) dont 4 familles sont absentes, alors que 3 familles sont fréquentes dans les 2 sites Katorwa et Nvomo avec une fréquence d'occurrence de $F \geq 25 \%$. II s'agit de la famille de Libelliidae, de Lumbricidae et de Pleidae. Le tableau 2 représente les différents signes de fréquence d'occurrence des familles récoltées au lac Vert. 

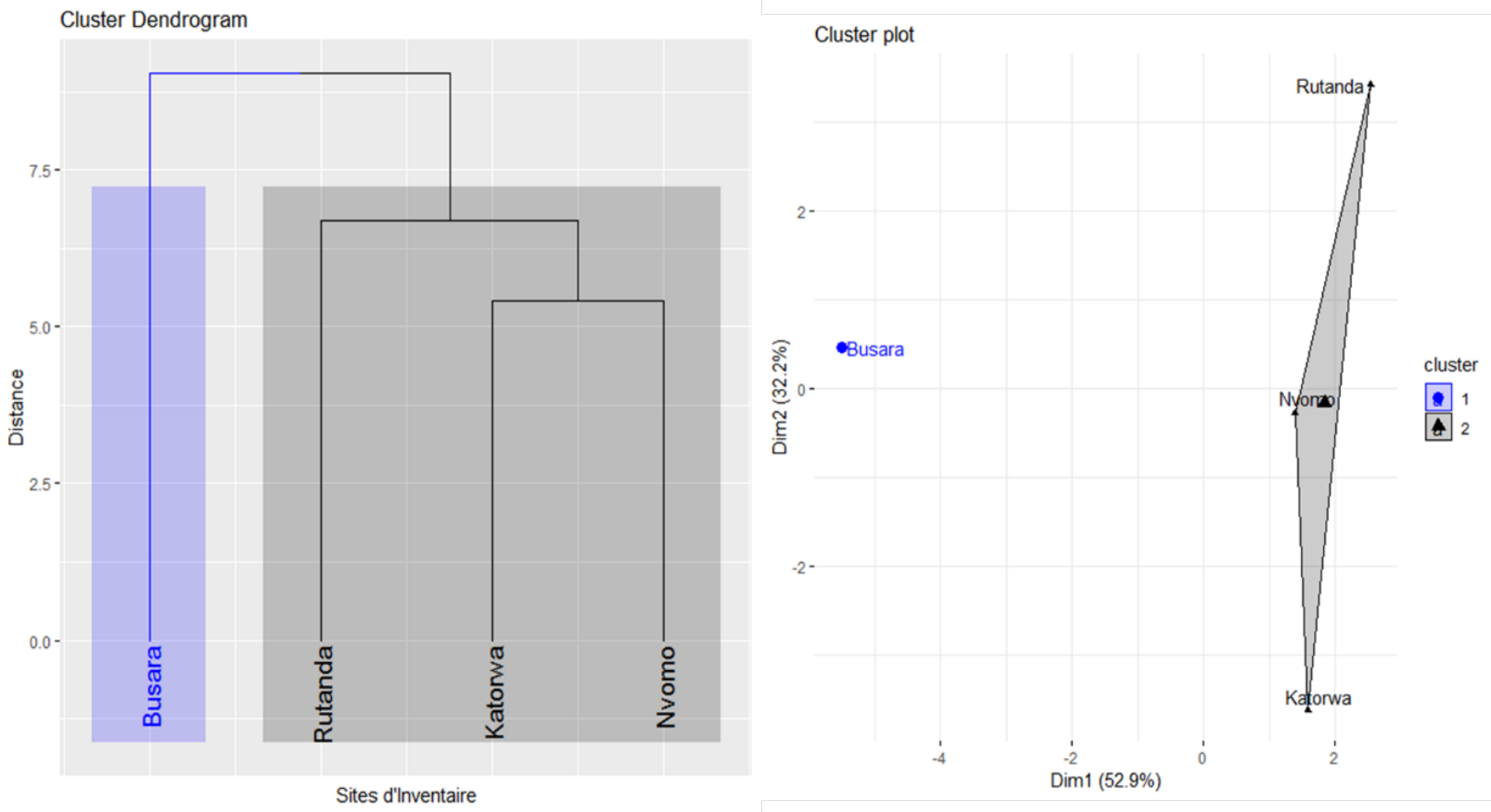

Figure : 2. Classification hiérarchique des sites du lac Vert basée sur la distance euclidienne

Tableau 2: Fréquence d'occurrence des différentes familles des macroinvertébrés aquatiques récoltés dans les sites du lac Vert.

\begin{tabular}{|c|c|c|c|c|}
\hline Familles & BUSARA & KATORWA & NVOMO & RUTANDA \\
\hline Limnaeidae & ++ & + & ++ & + \\
\hline Buccinidae & + & + & ++ & ++ \\
\hline Coenagrionidae & +++ & + & + & + \\
\hline Libelliidae & ++ & ++ & ++ & + \\
\hline Lestidae & + & + & +++ & - \\
\hline Aesnidae & +++ & - & + & - \\
\hline Chironomidae & + & +++ & + & + \\
\hline Tabanidae & - & +++ & - & - \\
\hline Syrphidae & ++ & - & ++ & ++ \\
\hline Lumbricidae & + & ++ & ++ & + \\
\hline Nepidae & ++ & + & + & ++ \\
\hline Hydrometridae & ++ & + & ++ & + \\
\hline Vellidae & ++ & ++ & + & + \\
\hline Corixidae & ++ & + & + & + \\
\hline Gerridae & ++ & + & ++ & + \\
\hline Belostomatidae & +++ & + & ++ & + \\
\hline Pleidae & ++ & ++ & ++ & + \\
\hline Distiscidae & +++ & + & + & + \\
\hline Capniidae & +++ & ++ & - & - \\
\hline Araneidae & +++ & - & + & + \\
\hline
\end{tabular}

- $\quad: F=0 \%$ (taxons absents)

$+\quad: F \neq 0 \%$ et inférieur à $25 \%$ (taxons rares)

$+\quad: 50 \%>F \geq 25 \%$ (taxons fréquents)

+++ : $50 \% \geq F$ (taxon très fréquents) 
S'agissant de genres, le site Busara présente 9 genres très fréquents avec une fréquence d'occurrence > à $50 \%$. Ces genres sont Enallagma, Erythroma, Aeshnia, Ranatra, Lethocerus, Elmis, Discus, Capioneura et Araneus. Les genres Chironomus et Tabanus sont très fréquents dans le site Katorwa. Dans le site Nvomo le seul genre de Lestes est très fréquent alors que le genre de Nepa est aussi très fréquent dans le site Rutanda. Les autres genres sont parfois rares ou fréquents dans les 4 sites d'échantillonnages. Le tableau 3 représente la fréquence d'occurrence des genres des macroinvertébrés aquatiques récoltés dans les sites d'échantillonnage au lac Vert.

Tableau 3: Fréquence d'occurrence des différents genres des macroinvertébrés aquatiques récoltés dans les sites d'échantillonnage du lac Vert.

\begin{tabular}{|c|c|c|c|c|}
\hline Genres & BUSARA & KATORWA & NVOMO & RUTANDA \\
\hline Stagnicola (Limnaeidae) & ++ & + & + & ++ \\
\hline Radix (Limnaeidae) & + & ++ & ++ & + \\
\hline Buccinilum (Buccinidae) & + & + & ++ & ++ \\
\hline Coenagrion (Coenagrionidae) & ++ & ++ & + & + \\
\hline Enallagma (Coenagrionigae) & +++ & + & + & + \\
\hline Erythroma (Coenagrionidae) & +++ & + & ++ & - \\
\hline Libellula (Libelliidae) & ++ & ++ & ++ & + \\
\hline Orthetum (Libeliidae) & ++ & ++ & + & - \\
\hline Lestes (Lestidae) & + & + & +++ & - \\
\hline Aeshnia (Aeshnia) & +++ & + & + & - \\
\hline Chironomus (Chironomidae) & + & +++ & ++ & + \\
\hline Tabanus (Tabanidae) & - & +++ & - & - \\
\hline Lumbricus (Lumbricidae) & + & ++ & ++ & + \\
\hline Ranatra (Nepidae) & +++ & + & + & ++ \\
\hline Nepa (Nepidae) & ++ & - & + & +++ \\
\hline Hydrometra (Hydrometridae) & ++ & + & ++ & + \\
\hline Microvelia (Vellidae) & ++ & ++ & + & + \\
\hline Micronecta (Corixidae) & ++ & + & + & + \\
\hline Aquarius (Gerridae) & ++ & + & ++ & + \\
\hline Lethocerus (Belostomatidae) & +++ & + & ++ & + \\
\hline Neoplea (Pleidae) & ++ & ++ & ++ & + \\
\hline Discus (Distiscidae) & +++ & ++ & ++ & + \\
\hline Acillus (Distiscidae) & ++ & + & + & ++ \\
\hline Elmis (Elmidae) & +++ & ++ & + & + \\
\hline Capioneura (Capniidae) & +++ & ++ & - & - \\
\hline Araneus (Araneidae) & +++ & + & + & + \\
\hline \multicolumn{5}{|c|}{ - $\quad: F=0 \%$ (taxons absents) } \\
\hline$+\quad: F \neq 0 \%$ et inférieur à 2 & ares) & & & \\
\hline$+\quad: 50 \%>F \geq 25 \%$ (taxons $f$ & & & & \\
\hline
\end{tabular}




\section{DISCUSSION}

Est-ce-que le fait que le Lac Vert n'a aucune communication directe en alimentation d'eau contient une diversité des macroinvertébrés aquatiques différente des autres écosystèmes aquatiques? Nombreuses études réalisées sur les macroinvertébrés aquatiques de lacs Tanganyika et Kivu par le P-BEATRA (2012), les écosystèmes lacustres du rift albertin par Yapo et al., (2014) et Marlier (1954) qui rapportent que les invertébrés sont les communautés les plus abondantes dans ces écosystèmes. Un nombre de 1653 individus récoltés dans les 4 sites d'échantillonnage répartis dans 10 ordres, 20 familles et 26 genres témoignent une bonne diversité du lac Vert. L'ordre le plus abondant est le Basomatrophore (Gastéropodes) rapproché par les caractéristiques des bioaccumulations et une chaine trophique des herbivores tels que Tachet et al. (2009) l'a prouvé. Au lac Vert, leur abondance serait due à la présence des périphyton et des macrophytes qui jouerait un rôle de filtreur des eaux. En RDC, Ferrari et al (1998) montre une diversité de 21 espèces des Gastéropodes (Basamatrophores, Neograstropodes) récoltés dans 229 stations et Baluku et al. (1989) identifient 7 espèces de Gastéropodes dans la région Est de la RDC où se situe le lac Vert. Nos résultats confirment les résultats de ces prédécesseurs où les caractéristiques limnologiques des lacs Kivu, Tanganyika et Edouard, diffèrent de ceux du lac Vert. La végétation riveraine et le faible dragage des berges $\mathrm{du}$ lac favoriseraient une abondance des ordres de Basamatrophores et Neogastropodes. Par ailleurs une abondance des insectes a été observée au lac entre autre les Odonates, les Coléoptères, les Hétéroptères et les Diptères ayant un caractère des prédateurs détritivores (Tachet et al., 2009) prouvant une bonne approche écologique favorable à leur développement préalablement à cause d'abondance des périphytoplanctons. D'où une diversité de ces genres dans tous les sites. Néanmoins, l'absence des Plécoptères dans les sites Nvomo et Rutanda fait allusion à l'absence du sédiment plus fin. Mary (2001) et Verneaux (1997) montrent qu'ils sont un bon indicateur de la qualité biologique des eaux car très sensibles aux perturbations de l'habitat. Leurs présences dans les sites Busara et Katorwa est que les activités anthropiques sont en régression. Dans un même angle d'idée, un projet similaire à notre recherche d'inventaire de la biodiversité des macroinvertébrés aquatiques des lacs Tanganyika et Kivu a été faite dans la région par le P-BEATRA (2012) qui montre une abondance de six ordres des macroinvertébrés aquatiques dans le bassin d'Uvira, 9 ordres dans le bassin de Lwiro. Tandis que Burgeon (1937) sur le lac Kivu a révélé une abondance de 3 ordres dont les Coléoptères, les Diptères et les Trichoptères. Cette étude est en relation avec les résultats de P-BEATRA (2012) en termes d'abondance des ordres liés aux caractéristiques écologiques du milieu et ne concordent pas avec ceux de Burgeon (1937) sur le lac Kivu. Le lac Vert a une absence de Trichoptères probablement suite à la rareté des mixages des eaux, et à la profondeur du lac. Les recherches de Ngera et al (2009a, 2009b) ; Zirirane et al (2014); Ndakala et al (2015) et Irenge (2012) ont testé différents sites dans la région du bassin du lac Kivu et ont montré que l'abondance et la diversité des macroinvertébrés aquatiques sont fonction de leurs exigences et tolérances éco-physiologiques. Nos résultats sont en corrélation avec ceux de Samba (2008), MDDP (2008), U.S. EPA (2012), Banaru et Perez (2010), Leclercq et al. (2010) qui ont décrit l'habitat des macroinvertébrés aquatiques et ses principales influences. Pour cette première étude d'inventaire préliminaire de macroinvertébrés, les observations des groupes présents dans les échantillons couplés à leur fréquence d'occurrence permettent de dégager des taxons de macroinvertébrés que l'on peut qualifier de polluo-sensibles. En effet, ces taxons sont seulement présents au niveau du site Busara avec des fréquences d'occurrence supérieures à 50\% ; ce sont: Coenagrionidae, Aeshnidae, Belostomatidae, Disticidae, Capniidae, et Areneidae. Les familles qui n'ont été rencontrées que dans les retenues anthropisées (Nvomo, Katorwa et Rutanda) peuvent être considérés comme des polluo-résistantes et pourraient être d'un autre type d'intérêt pour la bioindication de ces hydrosystèmes car leurs fréquences restent inférieures à $50 \%$. Le site protégé de Busara confirme son statut de station de référence avérée par la présence de Capniidae, cette famille d'Insectes avait été classée par Moisan et Pelletier (2008) comme taxon sensible au niveau des sites. La fréquence de la famille Tabanidae uniquement dans le site Katorwa peut être qualifiée d'une part son apport par un oiseau dans la région car ces individus sont présents dans le milieu d'étude. Le site Busara qui est le site témoin présente une fréquence élevée supérieur à $50 \%$ par rapport aux autres sites témoigné par ses caractéristiques de la végétation riveraine non influencé ainsi que les pierres comme substrats principaux. Ces 
paramètres étaient évalués par Palmer et al. (1997) où les peuplements des macroinvertébrés le long du gradient étaient influencés par la nature du substrat.

Les activités anthropiques sont-elles Relation entre activités anthropiques et indices écologiques des macroinvertébrés. : Les activités anthropiques n'ont pas d'influence significative sur la répartition des macroinvertébrés entre les sites d'échantillonnages cela n'a pas était le même cas pour la richesse spécifique où le site Busara a une différence significative par rapport au site Rutnada. Les 26 genres explorés dans le site témoin sont rapproché par le

\section{CONCLUSION}

Cette étude était axée sur l'inventaire préliminaire de macroinvertébrés aquatiques du lac Vert de la ville de Goma, Province du Nord-Kivu à l'Est de la République Démocratique du Congo. Dans cet écosystème situé dans la région du rift albertin, issu des activités tectoniques montre une abondance de 1635 individus récoltés dans les 4 sites choisis par rapport aux activités anthropiques, au type de substrat et à l'accessibilité. Une abondance des Coléoptères et Mollusques a été trouvés rapprochés par une richesse floristique importante cas des macrophytes et du phytoplancton. Les activités anthropiques sont les principaux facteurs influençant la diversité des macroinvertébrés. Ces derniers situent le lac Vert dans une même branche géomorphologique et contexte

\section{REMERCIEMENTS}

Nous remercions les agents de l'ICCN/Nord-Kivu pour son leur accompagnement en vue de l'accès au site. Que les animateurs du Département de Biologie et ceux du LPVMA-UOB de l'Université Officielle de

\section{BIBLIOGRAPHIE}

AFNOR, 2010. Qualité écologique des milieux aquatiques. Qualité de l'eau. Traitement au laboratoire d'échantillons contenant des macro-invertébrés des cours d'eau, Association Française de Normalisation; Prénorme expérimentale XP T90 - 333.

AFNOR, 992. Qualité écologique des milieux aquatiques, Qualité de l'eau, Détermination de I'Indice Biologique Globale Normalisé (IBGN), Association Française de Normalisation, Norme homologué T90-350.

Amiard, J., et Amiard-Triquet, C, 2008. Les biomarqueurs dans l'évaluation de l'état faible taux des activités anthropiques où l'agriculture n'est signalée que sur son bassin versant. Par contre le site de Rutanda est plus anthropisé de tous les sites par la lessive, la vaisselle, l'agriculture sur une pente forte ainsi que la présence des déchets plastiques à la surface des eaux. Ce paramètre est dans le même cas des observations des sites lors des récoltes des macroinvertébrés du bassin du lac Kivu par Ngera et al. (2009a, 2009b) ; Zirirane et al. (2014) ; Ndakala et al. (2015). Les activités anthropiques sont à prendre en considération dans l'évaluation de la diversité des macroinvertébrés aquatiques du lac Vert.

hydrologique des lacs du rift albertin. Les activités de reboisement sont à encourager pour la restauration de la biodiversité de ce Jardin Botanique et Zoologique. Par conséquent, les activités qui mettent ce site en péril comme l'exploitation de carrière de sable autour du lac, l'utilisation du site comme dépotoir sont à exclure par les gestionnaires du site. Ce travail n'est qu'un inventaire préliminaire de la biodiversité aquatique du lac Vert, les recherches taxonomiques de toute la biodiversité et les caractéristiques physicochimiques des eaux dans différentes strates feraient ce site un réel jardin zoologique et botanique qui attirerait non seulement les chercheurs mais aussi les touristes et les investisseurs.

Bukavu trouvent notre sentiment de gratitude pour leurs guides et apports pour l'amélioration de ce travail scientifique.

écologique des milieux aquatiques. Paris, Lavoisier, 400p.

Bagalwa, M., Karume, K., Bayongwa, C., Ndahama, N., Ndegeyi, K., 2012: Land-use Effects on Cirhanyobowa River Water Quality in D.R. Cong, Greener Journal of Environment Management and Public Safety, Vol. 1, num 1, 17-26pp.

Baguma, B.G., 2014. Contribution à l'étude des paramètres physico-chimiques et inventaire des macro-invertébrés benthiques de la rivière Nyamuhinga (Bukavu/RDC); TFC inédit UOB.

Baluku B., Josens G., Loreau M., 1989. Etude préliminaire de la densité et de la répartition 
des mollusques dans deux cours d'eaux du Zaïre oriental, Revue Zoologique Africaine, 103, 291-302.

Banaru, D., et Perez, T., 2010. Bioindicateurs, Biomarqueurs, Notes de cours. Université de Marseille, Paris, 24p.

Barbour, M.T., Gerritsen, J., Snyder, B.D., et Stribling, J.B., 1999. Rapid Bioassessment Protocols for Use in Wadeable Rivers: Periphyton, Benthic Macroinvertebrates, and Fish, $2^{\mathrm{e}}$ édition, Washington, D.C., U.S. Environmental Protection Agency, Office of Water, EPA 841B-99-002.

Beauger, A., 2008. Bioevaluation de la qualité de l'eau : établissement d'un protocole d'échantillonnage simplifié, basée sur la collecte des macroinvertèbrés benthiques sur les seuils des rivières à charge de fond graveleuse. Thèse, Université Blaise Pascal, 563p.

Belanger, D., 2009. Utilisation de la faune macrobenthique comme bio-indicateur de la qualité de l'environnement marin côtier, Mémoire de Maitrise, Université de Sherbrooke, Canada 46p.

Ben moussa A, Chahlaoui A, Rour E., Chahboune M., 2014 : Diversité taxonomique et structure de la macrofaune benthique des eaux superficielles de l'oued khoumane. Moulay idriss Zerhoun, Maroc (Taxonomic diversity and structure of benthic macrofauna of surface water of Khoumane River. Moulay idriss Zerhoun, Morocco) J. Mater. Environ. Sci. 5 (1) (2014) 183- ISSN : 2028-2508 CODEN : JMESCN 198

Boissonneault, Y., 2006. Le Suivi Ecologique des Rivières au QUEBEC: comparaison des bioindicateurs basés sur les invertébrés et les diatomées benthiques. Mémoire de maitrise, Université Trois-Rivières, 137p.

Burgeon, G., 1937: Liste des Coléoptères récoltés au cours de la mission Belge au Ruwenzori, Institut Royal Colonial Belge Section des Sciences Naturelles Et Médicales, Mémoires, Collection in- $8^{\circ}$, Tome VI, fascicule 1, Bruxelles, Librairie Falk fils, Georges Van Campenhout, Successeur, 22, Rue des Paroissiens, 22, 145p.

Cummins, K. W., 1975:Macroinvertebrates in Witthon, B.A. (eds), River Ecology, Studies in Ecology volume 2, Berkeley (Californie), University of California Press, 170-198pp.

CVRB, 2006. Suivi du macrofaune benthique: comparaison statistique d'outils d'identification des macro-invertébrés benthiques, Comité de Valorisation de la Rivière Beauport.

Dajoz, R.., 1982 : Précis d'écologie, 4e édition, Paris, Bordas, 503p.

De Pauw, N. et Vanhooren, G. 1983. Method for biological quality assessment of water courses in Belgium. Hydrobiologia. 100: 153 - 168.

Epler J. H., 2006. Identification manuel for the aquatic and semi-aquatic Heteroptera of Florida, Division of Water Ressource Management, Department of Environmental Protection, State of Florida, $195 \mathrm{P}$.

Ferreira, M., Wepener, V., and Van Viren, J.H., 1998: Aquatic Invertebrate Communities of perennial pans in Mpumalanga, South Africa: a diversity and function approach; Centre for Aquatic Research; Department of Zoology; Unisersity of Johannesburg; Auckland Park.

Gnohossou, P.M., 2006. La faune benthique d'une lagune ouest africaine (le lac Nokoué au benin), diversité, abondance, variations temporelles et spatiales, place dans la chaine trophique; Thèse de doctorant, Institut National Polytechnique de Toulouse, 184pp.

Grant I.F., 2016. Les Invertébrés Aquatiques; Natural Resources Institute, University of Greenwich at Medway, Central Avenue, Chatham Maritime, Kent ME4 4TB, R-U. Pg183-193.

Groga, N., 2012: Structure, fonctionnement et dynamique du phytoplancton dans le lac de Taabo (Côte d'Ivoire), Thèse de Doctorant, Université de Toulouse, 224pp.

Hammer, Ø. Harper, D.A.T., Ryan, P.D., 2001. PASTPalaeontological Statistics, version 2.6.

Hecky, R.E. \& Kling, H.J., 1987 : Phytoplankton ecology of the great lakes in the rift valleys of Central Africa. Arch. Hydrobiol. Beih. Ergebn. Limnol., $25: 197-228$.

HellawelL, J. M., 1986. Biological Indicators of Freshwater Pollution and Environmental Management, Londres, Elsevier, $546 \mathrm{p}$.

Hyangya B. 2013 : Essai d'évaluation de l'influence des activités anthropiques sur la physico-chimie, la composition et l'abondance du plancton et des macro-invertébrés du lac Tanganyika, Mémoire de Master en Environnement Aquatique, Université d'Abomey. 
Hynes, H. B. N., 1970. The Ecology of Running Waters, Toronto, University of Toronto, $555 \mathrm{p}$.

Hynes, H.B.N., 1960.The biology of polluted waters. Liverpool, University Press.

ICCN, 2014 : Plan d'aménagement du lac vert, Projet de mise en œuvre des stratégies pour la conservation du Jardin Zoologique et Botanique Lac Vert, Institut pour Congolais pour la Conservation de la Nature, 40p.

Irenge, E., 2012. Evaluation des qualités physicochimique et biologique des eaux de la rivière Nyamuhinga (Bukavu/RD-CONGO); Mémoire de Master inédit UEA.

Khettar, S., Haouchine, N., et Asselah, B., 2013. Macro invertébrés et qualité biologique de quelques cours d'eau du bassin versant de la Macta (ouest de l'Algérie), The $4^{\text {th }}$ International Congress Water, Waste \& Environment (EDE4) Agadir, Morroco.

Kwetu S. G., Karume K., Graba M. H., Mugaruka B. T., Kasereka M. C., Kyambikwa M. A., Kamate K. E., Kitumaini M. F., 2016. Contribution à l'étude géochimique des formations géologiques du volcan Nyiragongo: cas du cône du lac Vert à Goma, Nord-Kivu, R. D Congo; International Journal of Innovation and Applied Studies, ISSN 2028-9324, Vol. $15, N^{\circ} 3,685-696 \mathrm{pp}$.

Leclercq L., et Solite M., M., 2010: Clé simple de détermination des macroinvertébrés d'eau douce à l'usage de petit «gardien des rivières ", Station Scientifique des HautesFagnes; Université de Liège; $62 p$.

Leclerq L., 2001 : Intérêt et limites des méthodes d'estimation de la qualité de l'eau, Station Scientifique des Hautes-Fagnes, Belgique.

Léveque, C., 1996. Les écosystèmes aquatiques, Dépôt légal, 6907-08/96, Collection 50, Edition Paris, 15pp.

Marlier B 1954. Recherche hydro biologique dans les rivières du Congo Oriental.

Marry N., 2001. Revue de littérature sur la faune benthique d'eau douce de NouvelleCalédonie, Projet Kaniambo, P140.

MDDEP, 2008. Guide de surveillance biologique basée sur les macroinvertébrés benthiques d'eau douce $d u$ QUEBEC, Ministère $d u$ Développement Durable, de l'Environnement, et des Parcs. Québec, ISBN 978-2-55066035-4, 86p.
MECNT, 1997. Rapport intermédiaire sur la mise en œuvre de la convention relative à la diversité en République Démocratique du Congo, Ministère de l'Environnement, Conservation de la Nature et du Tourisme, 11-29 pp.

Melhaoui, M., et Berrahou, A., 2009. Echantillonnage et étude des macro-invertébrés de Moulaya du MAROC.

Micha, J.C., \& Noiset, J.L., 1982. Evaluation biologique de la pollution des ruisseaux et des rivières par les macro-invertébrés aquatiques, Revue, 51-142pp.

Millot G., 2009. Comprendre et réaliser les tests statistiques à l'aide de $\mathrm{R}$ manuel pour les débutants; Ed. De Boeck Université, ISBN 978-2-8041-0179-4; Pp14-170.

Moisan, J., et Pelletier, L., 2008.Guide de surveillance biologique basée sur les macro-invertébrés benthiques d'eau douce du Québec - Cours d'eau peu profonds à substrat grossier, 2008, Ministère du Développement Durable, de l'Environnement et des Parcs, ISBN : 978-2550-53591-1p 86.

Moussa, M. 2005. Les eaux résiduaires des tanneries et des teintureries: caractéristiques physicochimiques, bactériologiques et impact sur les eaux de surface et les eaux souterraines, Page 135.

Muhindo M. B., 2014 : Contextes géomorphologique et structural du cône volcanique du lac vert, Mémoire de Licence Inedit ULPGL/GOMA.

Ndakala, P.M., Bisimwa, A.M., Masilya, P.M., et Ngera, F.M., 2015. Etude du macrofaune aquatique de la rivière Kalengo, Sud-Kivu, République Démocratique du Congo, International Journal of Innovation and Scientific Research, ISSN 2351-8014 Vol. 13 No. 388-397pp.

Ngera, M.F., Baluku, B., Cammaerts, D. \& Bisimwa, M.A., 2009. Evaluation biologique de la pollution de la rivière Kalengo par les invertébrés aquatiques, Cahier du CERUKI, Numéro Spécial CRSN-Lwiro, p89-94

Ngera, M.F., Cammaerts, D., Bisimwa, M.A. \& Baluku, B., 2009. Étude comparative de macroinvertébrés benthiques de trois cours d'eau du bassin versant du lac Kivu en RD CONGO, Cahier du CERUKI, Numéro Spécial CRSNLwiro, p95-10

Nieser, N., 2004: Guide to aquatic heteroptera of Singapore and peninsular Malaysia III. Pleidae and Notonectidae; The Raffles Bulletin of 
Zoology 52 (1); National University of Singapore; 79-96.

P-BEATRA, 2012. Etude de la Biodiversité des Ecosystèmes du parc national de KahuziBièga (secteur de Tshibati) et du lac Tanganyika et trois de ses affluents à Uvira, Est de la République Démocratique du Congo, Rapport de la session de formation, Programme BEATRA.

Pollabauer, C., et Alliodo, R., 2010. Indice d'Intégrité Biotique (IIB) ; Les poissons, Indicateurs d'état des milieux aquatiques de Nouvelle Calédonie, ERBIO, Etudes et Recherches Biologique

Ramade, 2008. Dictionnaire Encyclopédique des sciences de la nature et de la biodiversité, DUNOD, Paris, 726.

Reyjol, Y., Vassilis, S., et Basilico, L., 2013. Bioindication : des outils pour évaluer l'état écologique des milieux aquatiques: Perspectives en vue du $2^{\mathrm{e}}$ cycle DCE - Eaux de surface continentales, 64p.Richards, C., R. J. Haro, L. C. Johnson \& G. E. Host, 1997.Catchment and reach-scale properties as indicators of macroinvertebrate species traits. Freshwater Biology 37:219-230.Rodier, J., 1984. L'analyse de l'eau : eaux naturelle, eaux résiduaires, eau de mer. 8 ème $\mathrm{Ed}$. Dunod. Paris, France.

Rosenberg, D., Davies, J., Cobb, D., Wiens, A., 1993. Protocoles de mesure de la biodiversité, Les macro-invertébrés benthiques dulcicoles. 44p.

Samba, 2008. Eau et Ecosystème Aquatique, des indicateurs intégrateurs, Journal Officielle, Paris, $15 p$.

Sanogo S., Kabre J. A. T., Cecchi P., 2014. Inventaire et distribution spatio-temporelle des macroinvertébrés bioindicateurs de trois plans d'eau du bassin de la volta au Burkina Faso, International Journal of Biological and Chemical Sciences, ISSN 1997-342X (on line), 25p.

Sarmento H., 2006 : Ecologie du phytoplancton du lac Kivu (Est Africa), Thèse de doctorat, Faculté des sciences, Département de Biologie, Université de Namur, 24-189pp

Tachet, H., Bournaud, M., Richoux, P., Dessaix et Pattee, 2009. Initiation aux invertébrés des eaux douces. Association Française de Limnologie, Université Lyon. Ecologie des Hydrosystèmes Fluviaux, Geolab, 199p.
Touzin, D., 2008. Utilisation des macro-invertébrés benthiques pour évaluer la dégradation de la qualité de l'eau des rivières au Québec, 52p.

U.S. EPA, 2002.Biological Assessments and Criteria: Crucial Components of Water Quality Programs, United States Environmental Protection Agency, EPA 822-F-02-006.

Vernaeux, J., 1973. Cours d'eau de Franche- comité (massif du Jura), Recherche écologique sur le réseau hydrographique du Doubs-essai biotypologie, Ed. CNRS ; 228p.

Véronique, B.C., 2014. Les facteurs de sélection des bio-indicateurs de la qualité des écosystèmes aquatiques : Elaboration d'un outil d'aide à la décision; Mémoire de maitrise. Université de SHEREBROOKE, 118p.

WWF., 2007. Macro-invertébrés-les habitants du fond de la rivière: importance des macroinvertébrés dans le cours d'eau, $21 \mathrm{p}$.

Yapo, M.L.; Atsé, B.C. and Kouassi, P. 2014: Composition, Abundance and Diversity of aquatic insect in fishponds of Southern Ivory Coast, West Africa;

Zirirane, D., Bagalwa, J.J., Isumbisho, M., Mulengezi, M., Mukumba, I., Bora, M., Mucheso, J.M., Lukamba, A., Iragi, Irenge, G., Kibangu, B.,F., et Kamangala, R., 2014. Évaluation comparée de la pollution des rivières Kahuwa et Mpungwe par l'utilisation des macroinvertébrés benthiques, International Journal of Innovation and Scientific Research, ISSN 2351-8014 Volume 14 Numéro 3. 